\title{
SLEEP APNEA AND REM SLEEP BEHAVIOR DISORDER IN PATIENTS WITH CHIARI MALFORMATIONS
}

\author{
Paulo Sérgio A. Henriques-Filho', Riccardo Pratesi ${ }^{2}$
}

\begin{abstract}
Background: Chiari malformations (CM) may result in the appearance of REM sleep behavior disorder (RBD) and sleep apnea syndrome (SAS) that can be considered markers of brain stem dysfunction. Purpose: To evaluate the frequency of RBD and SAS in patients with CM type I and II. Method: Were evaluated 103 patients with $\mathrm{CM}$ by means of full night polysomnography. Were scoring different sleep stages, frequency of abnormal movements (through video monitoring) and abnormal respiratory events. Results: Of the 103 patients, 36 showed CM type I and $67 \mathrm{CM}$ type II. Episodes of RBD were observed in 23 patients. Abnormal apnea-hypopnea index (AHI) was observed in 65 patients. Conclusion: The high rate of RBD suggests that this parassomnia and the increased frequency of central sleep apnea episodes, may be considered as a marker of progressive brain stem dysfunction.
\end{abstract}

KEY WORDS: Chiari malformation, polysomnography, parasomnia, REM sleep behavior disorder, RBD, sleep apnea syndrome, SAS, central sleep apnea, obstructive sleep apnea.

\begin{abstract}
Apnéia do sono e distúrbio do comportamento da fase do sono com REM em pacientes com malformações de Chiari

Resumo - Introdução: Malformações de Chiari (MC) podem gerar o aparecimento de distúrbio comportamental da fase do sono com REM (DCR) e síndrome da apnéia do sono (SAS), sugerindo a ocorrência de disfunção do tronco cerebral. Objetivo: Avaliar a frequêencia de DCR e SAS em pacientes com MC I ou II. Método: Utilizouse a polissonografia de noite inteira para a avaliação de 103 pacientes. Classificaram-se as diferentes fases do sono e analisou-se a frequeência de movimentos anormais (monitorada por vídeo) e de eventos respiratórios anormais. Resultados: Dos 103 pacientes analisados, 36 eram portadores de MC I e 67 de MC II. Episódios de DCR foram observados em 23 pacientes. $O$ índice de apnéia/hipopnéia foi considerado anormal em 65 pacientes. Conclusão: $A$ alta freqüência de DCR e o aumento da freqüência de episódios de apnéia central do sono podem ser considerados manifestação de disfunção progressiva do tronco cerebral.
\end{abstract}

PALAVRAS-CHAVE: malformação de Chiari, polissonografia, parassonia, distúrbio comportamental do sono REM, desordem respiratória do sono, apnéia central do sono, apnéia obstrutiva do sono.

Malformations characterized by herniation of the hindbrain through the foramen magnum were initially described by Chiari in 1891'. There are four types of Chiari malformation (CM), although the following two types are now recognized as being the most common: (a) Chiari malformation type I (CM I), that typically involves the isolated descent of the cerebellar tonsils into the cranio-vertebral junction resulting in a small posterior fossa and occasionally in hydrocephalus; (b) Chiari malformation type II (CM II), that is characterized by the descent of the inferior vermis and cerebellar hemispheres through the foramen magnum with a displacement of the brain stem (medulla, fourth ventricle, and lower portion of the pons) inside the spinal canal, resulting in aqueduct and fourth ventricle elongation. In this case, hydrocephalus is present in more than $70 \%$ of the cases ${ }^{1-3}$. Although CM I may cause cerebellar, brain stem or cervical symptoms because of impaction of the tonsils at the foramen magnum, and also due to disturbance of cerebrospinal fluid dynamics that may result in syringohydromyelia, an overt clinical picture with signs and symptoms of brain stem disorder is usually delayed until adult age. In contrast, in patients with the CM II, due to the greater complexity of their malformations, symptoms of brain stem dysfunction are generally present since birth ${ }^{4}$.

Although these manifestations are generally easily identified, some of them, such as the rapid eye movement sleep behavior disorder (RBD) and sleep apnea syn-

${ }^{1} \mathrm{MD}$, Graduation Program in Medical Sciences, Brasilia University School of Medicine, Brasilia, Brazil; ${ }^{2}$ MD, PhD, Senior Fellow Researcher.

Received 13 November 2007, received in final form 4 March 2008. Accepted 25 March 2008.

Dr. Paulo Sérgio A. Henriques-Filho - MILN TR 06, CH 232, C2, Lago Norte-71540-065 Brasilia DF - Brasil.E-mail: pazeredo@linkexpress.com.br 
drome (SAS) characterized by repeated events of partial or complete upper airways obstruction during sleep, having variable clinical presentations and being frequently sub-clinical, are more difficult to detect. RBD is a parasomnia characterized by the loss of normal muscle atonia during REM sleep with a concomitant disinhibition of mesencephalic motor pattern generators, resulting in phasic motor overactivity with behavioral release during REM sleep. This motor behavior, that is coincident with episodes of altered dreams of distinctly nightmarish content, is usually vivid, intense, action-packed out and violent. Since it occurs during a sleep phase that is normally associated with atonia, harmful or potentially harmful movements frequently result $t^{5,6}$. The exact incidence and prevalence of RBD are unknown because of inadequate reporting, but an overall prevalence of violent behaviors during sleep in the general population in the USA has been estimated at $0.5 \%{ }^{7,8}$. Although rarely reported, RBD can appear in malformations of the cranio-vertebral junction, eventually being the first sign of an undiagnosed $\mathrm{CM} \mathrm{I}^{9}$. In addition to RBD, patients with CM may exhibit a significant degree of sleep apneas and or hypoventilation. This sleep-disorder breathing (SDB) may presumably be caused by defects in the sensors (peripheral and central chemoreceptors) or by anatomic disruption of neurons and brain stem nuclei involved in central respiratory control. Mainly in patients with CM I, both RBD and SAS can be subclinical and remain undetected if not actively searched for. In this context, polysomnography can be used to establish the presence, type and severity of these sleep abnormalities, in CM patients ${ }^{10,11}$. Albeit references exist concerning the presence of RBD and SAS in malformations of the cranio-vertebral junction $^{9-12}$ as far as we know, no previous extensive study has been made focusing these sleep disorders among patients with $\mathrm{CM}$ I and II.

In the present study, we evaluated a series of $103 \mathrm{pa}-$ tients with CM types I and II utilizing full night polysomnography, in order to verify the frequency of RBD and SAS, and to determine the possible correlation with anatomical abnormalities. Since both RBD and SAS may be signs of brain dysfunction we expect that these data may be helpful in detecting unrecognized CM and establishing future measures for follow-up and management of these patients.

\section{METHOD}

\section{Subject selection}

Between March 2002 and March 2003 all patients seen at the outpatient clinic of the Sarah Rehabilitation Hospital of Brasilia due to varied complaints, and diagnosed as having CM, were consecutively admitted to the present study. The Sarah Rehabilitation Hospital pertaining to a nationwide multi-centre net of hospitals for the diagnosis and treatment of loco-motor system disorders. The diagnosis of CM was established on clinical grounds and on MRI findings. No patient had been referred for consultation specifically due to sleep related disorders although, upon questioning, history of restless sleep and nightmares with immediate dream recall, characteristic of RBD, was obtained from parents and/or caregivers, and from adult patients.

The study was approved by the Sarah Hospital Ethical Committee and informed consent was obtained from all participants or from their parents. Patients with epilepsy, patients using drugs that could interfere with their sleep patterns, and patients with brain lesions, or other malformations or abnormalities not related to the malformations under analysis were excluded from the study.

\section{Clinical interview}

Subjects admitted to the study underwent a complete history and physical examination. In addition, a comprehensive evaluation of their sleep characteristics was performed based on the International Classification of Sleep Disorders ${ }^{13}$.

\section{Sleep study}

Full night polysomnography evaluation was carried out utilizing a polygraphic digital system (Sleepscan Bio-Logical System Corporation, San Diego, CA, USA). Each subject underwent one or two full night polysomnography (depending on his or her adaptation to the sleep laboratory settings). Digital recordings included electroencephalogram (EEG) adopting identical montage (C3-A2, C4-A1, O1-A2 and O2-A1, according to the International 10-20 system), electro-oculogram, chin electromyogram (EMG,) electrocardiogram, upper airway sound, respiratory effort using piezoelectric belts over the chest and abdomen, and airflow through the nose and mouth (monitored through a cannula-pressure transducer and oronasal thermocouples). We also recorded bilateral surface EMG from the arms (biceps muscles) and legs (anterior tibial muscles). Oxy-haemoglobin saturation was monitored by pulse oximetry and subjects' behavior during sleep was monitored by continuous video and sound recordings. All patients disclosing any type of movement disorders during sleep or with history of possible RBD underwent EEG recordings with a complete 21 electrode montage.

\section{Scoring}

Sleep stages, patients' movements and abnormal respiratory events were scored by two accredited polysomnographic technologists according to the standard criteria of Rechtschaffen and Kales ${ }^{14}$. The REM sleep phase was scored separately in accordance with the method described by Lapierre and Montplaisir, and modified according to Sheldon and Jacobsen ${ }^{6}$, and Consens et al. ${ }^{15}:$ mini-epoch and epoch duration were respectively established as 3 and 30 seconds. The onset of a REM sleep period was determined by the presence of the first rapid eye movement 
Table 1. Density of phasic and tonic movements during total REM sleep time in 103 patients with and without RBD.

\begin{tabular}{lcccc}
\hline & CM I $(n=7)$ & CM II $(n=16)$ & All $(n=23)$ & \\
Patients with RBD & $33 \pm 1.7$ & $37.7 \pm 9.3$ & $36.8 \pm 8.6$ & $\mathrm{p}=0.03$ \\
& CM I $(\mathrm{n}=29)$ & CM II $(\mathrm{n}=51)$ & All $(\mathrm{n}=80)$ & \\
Patients without RBD & $21 \pm 2.7$ & $25.5 \pm 10.8$ & $23.9 \pm 8.9$ & \\
\hline
\end{tabular}

CMI, Chiari malformation type I; CMII, Chiari malformation type II; REM, rapid eye movement (sleep phase); RBD, REM sleep behavior disorder. Data are presented as mean \pm SD. A significance level of $p<0.05$ was used.

Table 2. PLMS index during total sleep time, during REM sleep, during episodes of arousal, and following SAS events in 103 patients with CM I and II.

\begin{tabular}{lccc}
\hline & CM I & CM II & \\
\hline PLMS/h during total sleep time & $21.3 \pm 36.6$ & $16.1 \pm 17.4$ & $\mathrm{p}=0.9$ \\
PLMS/h during REM sleep & $2.2 \pm 3.3$ & $12.5 \pm 4.8$ & $\mathrm{p}=0$ \\
PLMS/h with arousal episodes & $2.7 \pm 6.5$ & $2.6 \pm 1.6$ & $\mathrm{p}=0.87$ \\
PLMS/h following SAS events & $9.9 \pm 15.7$ & $0.7 \pm 0.7$ & $\mathrm{p}=0.06$ \\
\hline
\end{tabular}

PLMS, periodic limb movement during sleep; REM, rapid eye movement (sleep phase); SAS, sleep apnea syndrome. A significance level of $p<0.05$ was used.

and its termination identified either by the absence of REM during six consecutive epochs, or by the appearance of specific EEG features (K complexes, sleep spindles or signs of arousal). During the REM sleep phase, each 30-second epoch was scored and analyzed to establish the proportion of epochs showing a predominance of abnormally elevated background chin muscle tone (tonic component), and the proportion of 3-second mini-epochs (within the 30-second REM sleep epochs) showing bursts of EMG activity (phasic component). We utilized $30 \%$ of REM sleep epochs with abnormalities as our cut-off.

In children, the hypopnea and apnea duration cut-off utilized was two or more respiratory cycles ${ }^{16,17}$. The length of central apneas $\geq 10$ s were quantitated, as abnormal. Central apneas of any length that were associated with desaturation $\geq 4 \%$ from the average Spo2. These events were only considered significant when associated to a oxy-haemoglobin desaturation equal to or greater than $4 \%$ and/or with arousal, partial arousal or awakening ${ }^{18}$. The total duration of arterial desaturation was quantified as the cumulated span of arterial oxyhemoglobin saturation below $90 \%$, because below this level, like an arterial oxygen saturation of $85 \%$, due to concerns about reduced delivery of oxygen to the tissues ${ }^{19}$.

The apnea-hypopnea index (AHI) was defined as the sum of the equal types of apneic and hypopneic episodes per hour (obstructive, mixed or central). AHI were evaluated according to the patient's age. In adults, $\mathrm{AHI}$ indices values were considered mildly abnormal between 5 and 15 episodes per hour, moderately abnormal between 16 and 30 episodes, and severely abnormal when 30 episodes per hour were presented. In children and adolescents, between 1 and 5 episodes per hour were considered mildly abnormal, between 6 and 10 as moderate, and above 10 as severely abnormal ${ }^{12}$.
Periodic limb movements were scored according Zucconi et al classification ${ }^{20}$.

\section{Statistical analysis}

The statistical analysis was performed using SPSS software for Windows version 10.1 (SPSS, Chicago, IL) ${ }^{21}$. Sleep variables showing normal distribution were expressed as mean value \pm standard deviation. Sleep variables showing an abnormal distribution were expressed as median values (range and $10^{\text {th }}$, $25^{\text {th }}, 75^{\text {th }}$ and $90^{\text {th }}$ percentiles). Differences in continuous variables were tested by one-way ANOVA. The relationship between pairs of continuous variables was tested by Pearson correlation coefficients and linear regression. The Fisher test was utilized to determine association between discreet variables. A value of $\mathrm{p}<0.05$ was regarded as significant.

\section{RESULTS}

Of the 103 patients analyzed, 36 (mean age: $38 \pm 12.5$; age range: 9 to 70 years) showed CM type I and 67 (mean age: $7 \pm 4$; age range: 2 to 21 ) disclosed CM type II.

Episodes of RBD were observed in 23 patients, six being affected by CM I and 17 by CM II. In 10 of these patients RBD, was the only observed abnormality. During REM sleep all 23 RBD patients showed several types of abnormal motor behavior that were directly observed and registered by the video monitoring system, along with an observed increase in the density of phasic and tonic movements (Table 1). There was not SAS related to RBD.

PLMS index during total sleep time, during total REM sleep phase, with arousal and following SAS events can be seen in Table 2. 
Table 3. Comparison of the index of periodic limb movement during sleep (PLM) between the groups of CMI and CMII patients with and without RBD during total sleep time, during REM sleep, during episodes of arousal, and following apneas/hypopneas.

\begin{tabular}{|c|c|c|c|c|c|c|}
\hline & \multicolumn{3}{|c|}{ CMI } & \multicolumn{3}{|c|}{ CMII } \\
\hline & With RBD & Without RB & & With RBD & Without RBD & \\
\hline PLMS/h during total sleep time & $11.1 \pm 16.6$ & $3.65 \pm 8.5$ & $P=0$ & $8.2 \pm 16.1$ & $11.3 \pm 25.3$ & $\mathrm{p}>0.05$ \\
\hline PLMS/h during REM sleep & $2.3 \pm 3.5$ & $1.4 \pm 4.2$ & $\mathrm{P}=0.05$ & $3.9 \pm 10.2$ & $1.2 \pm 4.8$ & $\mathrm{p}>0.05$ \\
\hline PLMS/h with arousal & $0.4 \pm 1$ & $1.9 \pm 4$ & $P=0.37$ & $2 \pm 6.9$ & $0.4 \pm 0.1$ & $p=0.37$ \\
\hline PLMS/h following apnea/hypopnea & $3.9 \pm 10$ & $0.1 \pm 0.3$ & $P=0.03$ & $0.1 \pm 0.6$ & 0 & $p>0.05$ \\
\hline
\end{tabular}

PLMS, periodic limb movement during sleep; REM, rapid eye movement (sleep phase); SAS, sleep apnea syndrome. A significance level of $p<0.05$ was used.

Table 4. Index of the apnea/hypopnea, desaturation and snoring per hour during total sleep time in patients with CM I and II.

\begin{tabular}{lccc}
\hline & CM I (n=36) & CM II (n=67) & $\mathrm{P}$ \\
\hline AHI/h of sleep & $10.72 \pm 16.03$ & $4.89 \pm 15.03$ & 0.012 \\
Desaturation Index/h of sleep & $12.47 \pm 18.55$ & $8.98 \pm 14.79$ & 0.033 \\
Snore/h of sleep & $6.71 \pm 15.42$ & $0.76 \pm 4.63$ & 0.027 \\
Desaturation Below 90\%/h of sleep & $3.23 \pm 3.72$ & $2.78 \pm 6.02$ & 0.84 \\
\hline
\end{tabular}

$\mathrm{AHI} / \mathrm{h}$, apnea/hypopnea index, number of apnea and or hypopnea per hour; desaturation below $90 \% / \mathrm{h}$, number of desaturation events per hour, below $90 \%$, level of begin of impairment of haemoglobin dissociation; snore $/ \mathrm{h}$, number of snore per hour. A significance level of $p<0.05$ was used.

A comparison of the PLMS index between the group of 23 patients with RBD and the group of 80 patients, without RBD can be seen in Table 3.

Abnormal AHI was observed in 65 patients (65/103; $63 \%), 13$ of them (13/103; 10\%) being concomitantly affected by RBD. Among the 65 patients with abnormal AHI, 61 showed central sleep apnea $(61 / 65 ; 94 \%)$, one (1/65; $1,5 \%)$ disclosed obstructive sleep apnea, and three ( $3 / 65$; $4,5 \%$ ) presented both central and obstructive sleep apnea. Only one among the 23 patients with RBD showed severe sleep apnea.

The lack of an esophageal pressure transducer precluded analysis of the UARS.

The index of the abnormal apnea/hypopnea events, episodes of oxy-haemoglobin desaturation and episodes of snoring during the total sleep time of the 103 patients can be observed in Table 4.

Sixteen of the 65 patients with abnormal polysomnography findings disclosed sleep efficiency below $85 \%$. The worse sleep efficiency was observed among the 13 patients that showed both RBD and abnormal AHI. The wake time after sleep onset (WASO), in percent values, in CMl patient was $25.41 \pm 20.64$, and in CMII patients was $13.82 \pm 17.64$. The sleep efficiency, either in percent values, was in CMI patients $77.64 \pm 13.51$, and in CMII patients $85.78 \pm 15.41$.

All subjects showed normal body mass indexes (BMI: CMI - 22.23 \pm 9.03 ; CMII - 17.29 \pm 6.29 ) besides three pa- tients who were overweight. No correlation could be established between an increased BMI and the presence of abnormal AHI.

\section{DISCUSSION}

Our sample was exclusively comprised of patients with CM I and CM II. The complete absence of patients with CM III and IV can probably be explained by the increased complexity of the first and the generally subclinical presentation of the second. Patients affected by CM III and IV are seldom seen at tertiary level hospitals.

RBD has been also described as being associated with narcolepsy, agrypnia excitata, multiple sclerosis, ischemic or hemorrhagic cerebrovascular diseases and cerebellopontine tumors, as well as other disorders that affect brain stem REM sleep generators ${ }^{22-24}$. Of the 103 patients included in this study, 23 were affected by RBD. The high prevalence of RBD found among our patients is probably due to the fact that our sample was solely comprised of patients with CM. The first description of the relation between CM and RBD was made of a patient described by Lapierre and Montplaisir'. RBD in CM patients may be related to brain stem disgenetics abnormalities, because they suffer from traumatic chronic bone structure injuries, due to growth-related stretching ${ }^{5,25,26}$. These brain stem injuries will provoke abnormal movements, muscle tonus elevation, REM density elevation, and electromy- 
ography phasic activity elevation during the REM sleep phase, which may be explained by the dysfunction of the groups of neurons responsible for inhibitory motor functions during the REM sleep phase. These neurons are represented by the "pontis oralis", "pontis caudalis" and magnocelular nucleous, located in the reticular formation in the pons tegment. There is another subgroup with the same function, the subgroup alpha of the locus coeruleus located in the ventral and medial region of the medulla oblongata, which could be related to this brain stem dysfunction $^{27-29}$.

The analysis of the mean values of the density of phasic and tonic movements during total REM sleep time, in patients with and without RBD, showed that almost all values for patients with RBD (Table 1) were above those normally considered normal cut-off values ${ }^{15}$. This abnormality is probably consequent to a disruption of cortical and medullary neurons due to dysgenetic abnormalities, syringomyelia, or syringobulbia, which were frequently ascertained by the MRI findings.

Another abnormality observed in RBD patients with CM I and CMII as compared to patients with CM I and CMII without RBD (Table 2, Table 3) was a higher PLMS index in the former, which was a finding of exam. This statistically significant difference is probably related to a greater complexity of the brain and brain stem abnormalities found in patients with CM I and CMII with RBD'. In all patients with increased PMLS indexes, the MRI studies indicated hydrosyringomyelia and syringobulbia with an abnormal signal in $\mathrm{Tl}$ in the inferior region of the brain stem ${ }^{30}$.

The finding of abnormal $\mathrm{AHI}$ in 13 of 23 patients with RBD calls attention to a possible association between abnormal respiratory events and RBD. Abnormal respiratory events have been previously described in $\mathrm{CM}^{10,27,31}$ but there is poor information regarding the associate presence of RBD in this disorder and, as far as we know (aside from the isolated case reported by Lapierre and Montplaisir? that suggested the dysfunction of lower brain stem neural structures responsible for muscle atonia as a possible cause), RBD has not been previously mentioned as a possible consequence of CM. Sforza et al..$^{24}$, in an extensive review of RBD clinical and physiopathological findings, stated that the association between sleep apnea and RBD is uncommon, and when present, the apneas are essentially of the obstructive type. This assertion is not coincident with our findings in which thirteen patients disclosed both RBD and sleep-disordered central breathing and only one among them was affected by obstructive sleep apnea. The difference with results obtained by Sforza et al. ${ }^{24}$ may probably be explained by the different anatomical localization of the neuronal nuclei responsible for this kind of sleep apnea and the nuclei responsible for RBD, in patients with neurodegenerative disorders. This is not the case for patients suffering from $\mathrm{CM}$ in which these abnormalities are mainly dependent on a broad and variable degree of disruption of the neural structures in the medulla. Generally, this dysfunction can be ameliorated by surgery ${ }^{31}$. As an example, one of our CM I patients, who disclosed RBD and severe central sleep apnea, significantly improved after undergoing neurosurgical enlargement of the occipital region of the skull which led to a decreased frequency of the sleep apneas and RBD episodes. Improvement of brain stem functions, in this particular case, reinforces the hypothesis that, as previously stated, injuries caused by chronic brain stem compression are partially responsible for sleep abnormalities in CM patients.

Further review of the physiopathology of $\mathrm{CM}$ and brain stem anatomy may contribute to better understand the origin of the relation between RBD and SAS. The association of RBD and sleep apnea syndrome found in 13 of our patients (two with CM I, and 11 with CM II) can be explained by the proximity, in the brain stem, of neuronal groups responsible for the control of breathing and the control of REM sleep. REM sleep control centers and some of the respiratory control centers are situated between the caudal region of the facial nucleus and the ambiguous nucleus located in the lateral tegument of the gigantocelular region of the medulla. These tonically drive the respiratory system during REM sleep and, when damaged, are responsible for inspiratory central apneas ${ }^{28,29,31}$.

Among patients with $\mathrm{CMI}$ and $\mathrm{CMII}$, a higher rate of incidence of $\mathrm{AHI}$ was noticed for patients with $\mathrm{CMI}$ than for those with CMII (Table 4), since the CMII patients were mainly children. However, regarding severity, both were predominantly mild, with upper the limit for CMII patients being moderate. The increased severity of $\mathrm{AHI}$ among CMIl patients may be explained by the greater complexity at birth of this type of malformation, in comparing with the $\mathrm{CMI}$.

In comparing the sleep structures between individuals with CMI and CMII, greater fragmentation may be observed, with lower sleep efficiency percentages, among the CMI patients. These alterations are probably being linked to the greater age of the $\mathrm{CMI}$ patients in relation to the CMII patients, since the sleep efficiency cut off point is lower for children, and the micro-arousal rate is lower than in adults ${ }^{18}$.

The natural history of craniocervical transition pathologies has not been completely elucidated, but evidence exists that their accompanying abnormalities may show a progressive worsening ${ }^{31}$. It is also been stated that an augmented $\mathrm{AHI}$ is seen concomitantly with the progressive 
worsening of these pathologies ${ }^{31}$. Along the same line of reasoning, it would be expected that the appearance of RBD episodes or an increase in their frequency in patients with CM could herald a worsening of pre-existing lesions.

In conclusion, the relatively high rate of RBD among our CM patients and increase in the frequency of central sleep apnea episodes suggests the presence of progressive brain stem dysfunction. In this context, full-night polysomnography may be used as a follow-up instrument to analyze brain stem function in CM patients.

ACKNOWLEDGMENTS - The authors would like to thanks David Haxton Jr for the English revision of the final version of this paper.

\section{REFERENCES}

1. Koehler PJ. Chiari's description of cerebellar ectopy (1891). With a summary of Cleland's and Arnold's contributions and some early observations on neural-tube defects. J Neurosurg 1991;75:823-826.

2. Cai C, Oakes WJ. Hindbrain herniation syndromes: the Chiari malformations (I and II). Sem Pediatr Neurol 1997:4:179-191.

3. Gilbert JN, Jones KL, Rorke LB, Chernoff GF, James HE. Central nervous system anomalies associated with meningomyelocele, hydrocephalus, and the Arnold-Chiari malformation: reappraisal of theories regarding the pathogenesis of posterior neural tube closure defects. Neurosurgery 1986;18:559-564.

4. Salomão JF, Bellas AR, Leibinger RD, Barbosa AP, Brandão MAPB. Malformação de Chiari tipo II sintomática. Arq Neuropsiquiatr 1998;56:98-106.

5. Schenck $\mathrm{CH}$, Mahowald MW. REM sleep behavior disorder: clinical, developmental, and neuroscience perspectives 16 years after its formal identification in SLEEP. Sleep 2002;25:120-138.

6. Sheldon $\mathrm{SH}$, Jacobsen J. REM-sleep motor disorder in children. J Child Neurol 1998;13:257-260.

7. Udin $\mathrm{AB}$, Jarmi T. REM sleep behavior disorder. eMedicine [serial online] Available from: URL http:/ / www.emedicine.com/neuro/topic524. $\mathrm{htm}$. Acessado em 10/2006.

8. Ohayon MM, Caulet M, Priest RG. Violent behavior during sleep. J Clin Psychiatry 1997;58:369-376.

9. Lapierre O, Montplaisir J. Polysomnographic features of REM sleep behavior disorder: development of a scoring method. Neurology 1992;42:1371-1374.

10. Botelho RV, Bittencourt LR, Rotta JM, Tufik S. Polysomnographic respiratory findings in patients with Arnold-Chiari type I malformation and basilar invagination, with or without syringomyelia: preliminary report of a series of cases. Neurosurg Rev 2000;23:151-155.

11. Botelho RV, Bittencourt LR, Rotta JM, Tufik S. A prospective controlled study of sleep respiratory events in patients with craniovertebral junction malformation. J Neurosurg 2003;99:1004-1009.
12. Waters KA, Forbes P, Morielli A, et al. Sleep-disordered breathing in children with myelomeningocele. J Pediatr 1998;132:672-686.

13. International Classification Of Sleep Disorders: Diagnostic And Coding Manual, revised, MN: American Sleep Disorders Association; 1997.

14. Rechtschaffen A, Kales A. A manual of standardized terminology; techniques and scoring system for sleep stages of human subjects. Los Angeles: UCLA Brain Information Service / Brain Information Institute, 1968

15. Consens FB, Chervin RD, Koeppe RA et al. Validation of a polysomnographic score for REM sleep behavior disorder. Sleep 2005;28:993-997.

16. Uliel S, Tauman R, Greenfeld M, Sivan Y. Normal polysomnography respiratory values in children and adolescents. Chest 2004;125:872-878.

17. Verhulst SL, Schrauwen N, Haentjens, Gaal V, De Backer W A, Desager $\mathrm{KN}$. Reference values for sleep-related respiratory variables in asymptomatic European children and adolescents. Pediatr Pulmonol 2007; 42:159-167.

18. Carskadon M, Rechtschaffen A. Monitoring and staging human sleep. In Kryger MH, Roth T, Dement WC (Eds). Principles and practice of sleep medicine. $3^{\text {rd }}$ Edition. Philadelphia: WB Saunders, 2005:1359-1377.

19. Beasley R, McNaughton A, Robinson G. New look at the oxy-haemoglobin dissociation curve. Lancet 2006;367:1124-1126.

20. Zucconi M, Ferri R, Allen R, et al. The official World Association of Sleep Medicine (WASM) standards for recording and scoring periodic leg movements in sleep (PLMS) and wakefulness (PLMW) developed in collaboration with a task force from the International Restless Legs Syndrome Study Group (IRLSSG). Sleep Medicine 2006;7:175-183.

21. SPSS for Windows. Standard version. Release 10.0.1. SPSS Inc.1999.

22. Abad VC, Guilleminault C. Review of rapid eye movement behavior sleep disorders.Curr Neurol Neurosci Rep 2004;4:157-156.

23. Mahowald MW, Schenck CH. Rem sleep without atonia-from cats to humans. Arch Ital Biol 2004;142:469-478.

24. Sforza E, Zucconi M, Petronelli R, Lugaresi E, Cirignotta F. REM sleep behavioral disorders. Eur Neurol 1988;28:295-300.

25. Schijman E, History, anatomic forms, and pathogenesis of Chiari I malformations.Childs Nerv Syst 2004;20:323-328.

26. Culebras A, Moore JT. Magnetic resonance findings in REM sleep behavior disorder. Neurology 1989;39:1519-1523.

27. Zolty P, Sanders MH, Pollack IF. Chiari malformation and sleep-disordered breathing: a review of diagnostic and management issues. Sleep 2000;23:637-643.

28. Lai YY, Siegel JM. Medullary regions mediating atonia. J Neurosci 1988; 8:4790-4796.

29. Hendricks JC, Morrison AR, Mann G L. Different behaviors during paradoxical sleep without atonia depend on pontine lesion site. Brain Res 1982;239:81-105.

30. Nogues MA, Gene R, Encabo H. Risk of sudden death during sleep in syringomyelia and syringobulbia. J Neurol Neurosurg Psychiatry 1992;55:585-589.

31. Gagnadoux F, Meslier N, Svab I, Menei P, Racineux JL. Sleep-disordered breathing in patients with Chiari malformation: improvement after surgery. Neurology 2006;66:136-138. 